\title{
Enhanced Convolution Approach for Connection Admission Control in ATM Networks
}

\author{
J.L. Marzo *, J. Domingo-Pascual **, R. Fabregat *, \\ J. Solé-Pareta **
}

* Institut d'Informàtica i Aplicacions. Universitat de Girona Avda Lluís de Santaló s/n, 17071Girona (Spain).

Phone +34 972418475 FAX +34972 418399

e-mail \{marzo, ramon] @eia.udg.es

**Departament d'Arquitectura de Computadors. Universitat Politècnica de Catalunya. Gran Capita, s/n. Modul D6 Campus Nord 08071 Barcelona (SPAIN)

Phone: +34-3-4017001 Fax: +34-3-4017055

e-mail \{jordid,pareta\}@ac.upc.es

\begin{abstract}
In this paper the utilisation of the Probability of Congestion (PC) as a bandwidth allocation decision parameter is presented. We assume short buffers at the switch nodes to cope with cell level multiplexation contention ("bufferless" environments). Therefore, delay and cell delay variations are strongly bounded. Moreover, the Cell Loss Ratio (CLR) becomes the critical performance parameter. The validity of PC utilisation is compared with quality of service parameters in bufferless environments. The convolution algorithm is an accurate approach for Connection Admission Control (CAC) in ATM networks with small buffers. However, the convolution approach has a considerable computation cost, in terms of calculation and memory. To overcome these drawbacks, a new method of evaluation is proposed and analysed: the Enhanced Convolution Approach (ECA). In complex scenarios, with ECA, PC calculation can be carried out in real time while maintaining the desired accuracy. Several experiments have been carried out
\end{abstract}

The original version of this chapter was revised: The copyright line was incorrect. This has been corrected. The Erratum to this chapter is available at DOI: $10.1007 / 978-0-387-35388-3 \_42$ 
to compare the demanded bandwidth evaluated by: analytical methods, simulations and measurements in actual ATM switches. The main contribution of this paper is the proposal and analysis of ECA to the PC-evaluation for use in CAC schemes

\section{Keywords \\ Connection Admission Control, Traffic Management, B-ISDN and ATM}

\section{INTRODUCTION}

The Asynchronous Transfer Mode (ATM) transport network is based on fast packet switching using small fixed-size packets called cells. ATM permits flexible bandwidth allocation, so an important objective is to obtain the maximum statistical gain on a shared resource: the physical link. However, the bursty nature of the ATM traffic imposes strict requirements for traffic control. This paper is focused on the utilisation of Connection Admission Control as preventive control method to real time services.

Call Admission Control (CAC) is a procedure responsible for determining whether a connection request is admitted or denied. The procedure is based on resource allocation schemes applied to each link and switching unit. CAC schemes may be classified as non-statistical allocation (peak allocation) and statistical allocation, this paper relates to the second case. In statistical allocation, bandwidth for a new connection is not allocated on the basis of peak rate; rather the allocated bandwidth is less than the peak rate of the source (the sum of peak rates may be greater than the capacity of the output link). The determination of a simple and efficient CAC policy is one of the major challenges in the design and implementation of an ATMbased B-ISDN.

The maximum statistical multiplexing gain can be achieved if the network knows the probability distribution density function of the individual sources. The network needs a complete characterisation of sources with a known behaviour in statistical terms. A set of standardised parameters describes the behaviour of VBR connections in statistical terms. This parameters are: Peak Cell Rate (PCR), Sustainable Cell Rate (SCR) and Burst Tolerance (BT). The VBR bearer capability can be partitioned in two types: a) real-time (rt-VBR) that requires tightly constrained delay and delay variation, (as voice and video interactive applications), and b) non-real-time (nrt-VBR) where only a maximum cell transfer delay is considered (e.g. data transmissions with QOS guaranteed). This paper is mainly focused on CAC aspects relating to (rt-VBR) traffic management.

Adequate traffic characterisation is required to properly design and operate the ATM network, but the wide range of possible future services make this task very complex (Kleinvewillinghöfer, 1991). Inevitably, any characterisation of traffic must be in terms of the specific times at which cells are generated by the traffic source.

\section{BANDWIDTH ALLOCATION IN ATM NETWORKS}

\subsection{Previous Work}

The exact evaluation of the possible connections onto a link, maximising the statistical multiplexing gain with guaranteed QOS, is a difficult aspect in ATM 
networks management (Castelli, 1991), (Hui, 1988), (Bolla, 1997) and (Ohta, 1992). This is due to QOS parameters dependencies: assigned bandwidth and buffer size in a link.

J. Hui proposed a multilevel congestion and control model mechanism in (Hui, 1988). This model defines three different levels: the cell (packet), burst and call level. Those levels are based on the behaviour of the integrated traffic. Different statistical parameters are required to define the traffic at each level.

With reference to the QOS parameter CLR, it can be analysed in both, cell (CLRc) and burst (CLRb) levels. CLRb (burst) is the dominant factor for large buffers and CLRc (cell) is the dominant factor for small buffers (Castelli, 1991), (Handel, 1994) and (Miyao, 1993). It is very interesting to analyse environments with buffers large enough to make CLRc negligible, but small enough to trail the approximation for CLRb close to a bufferless model. This relevant aspect is further detailed in this work.

It is possible to assign an equivalent bandwidth (effective bandwidth for some authors) to each source that reflects its characteristics. The notion of "effective" bandwidth for each connection aims to summarise in a single parameter the bandwidth and QOS requirements of a connection. At the burst level, two different approaches for equivalent bandwidth evaluation are studied by (Guerin, 1991) and (Gallasi, 1989), in which different aspects of the behaviour of multiplexed connections are considered and fluid-flow model and stationary bit-rate distributions are presented. The fluid-flow model is also studied by (Castelli, 1991).

The fluid-flow model estimates the equivalent bandwidth when the individual impact of connections is critical. This model does not consider any multiplexing aspect. The fluid-flow model assumes that the information arrives uniformly during a burst and that the server removes the information from the queue in the same manner. Yang and Tsang in (Yang, 1995) describe an approach to estimate the cell loss probability for traffic scenarios with identical traffic sources (homogeneous traffic).

Stationary approximations: In this case the effect of statistical multiplexing is the dominant factor, and it considers that cells are lost when the instantaneous rate is greater than the bandwidth provided by the link. Small buffers are not effective at the burst level. Three methods are introduced below.

Binomial: the distribution of the aggregate bit rate on a link can be determined from the stationary distribution of the Markov chain formed by the superposition of sources.

Gaussian: this scheme (also referred to as both the normal approximation and the two-moment allocation scheme) assumes the independence of the traffic behaviour of the connections and characterises the multiplexed traffic by a normal distribution. The sum of the means and the sum of the variances of each connection give these parameters. A connection is only accepted if the congestion probability derived from the tail of the normal distribution is less than a pre-specified threshold. The Gaussian assumption is not applicable when there are small numbers of very bursty connections with high peak rates, low utilisation, and long burst periods. 
Convolution: the exact distribution of the aggregate bit rate can be determined by convolution using the exact bandwidth requirements of each traffic type. This method is based on the formula:

$$
\mathrm{P}(\mathrm{Y}+\mathrm{X}=\mathrm{b})=\sum_{\mathrm{k}=0}^{\mathrm{b}} \mathrm{P}(\mathrm{Y}=\mathrm{b}-\mathrm{k}) \mathrm{P}(\mathrm{X}=\mathrm{k})
$$

$\mathrm{X}$ and $\mathrm{Y}$ refers to the bandwidth requirement of the new connection and of the already established connections respectively; and $\mathrm{b}$ denotes the instantaneous required bandwidth. The above expression allows the evaluation of the distribution function of the demanded bandwidth on a link; this method is explained in detail in section 4.1.

The Decision Criterion in order to accept a new connection $\mathrm{X}$ when convolution is used in CAC is based on the Probability of Congestion (PC):

$$
\mathrm{PC}(\mathrm{Y}+\mathrm{X})=\mathrm{P}([\mathrm{Y}+\mathrm{X}]>\mathrm{C})=\sum_{b>C} P(Y+X=b)<\varepsilon
$$

Heuristic Methods: the other group of CAC approaches is based on heuristics and data modelling techniques (Saito, 1992). The neural network and fuzzy logic based approaches are example of this kind of approaches. Heuristic approaches provide a mechanism for clustering data obtained from ATM traffic measurements in a structure that constitutes the traffic model. A common example is a net structure composed of a set of neurones and respective connections for neural nets and a rule structure composed of a set of "if-then" associations of variables in the case of fuzzy systems (Ramalho, 1994).

\subsection{Drawbacks}

Several limitations have been found in the previous studies: Inter-dependencies: all studied models describe the behaviour of the sources without considering their interactions inside the network. The feasibility of performance objectives in ATM networks with correlated traffic is also studied in (Hee, 1993). Heterogeneous environments: normally, the performance of the evaluation approximations loses accuracy in heterogeneous scenarios. In these environments there are a trade-off between the Integration (Complete Sharing) vs. Segregation (Complete Partitioning) approaches. Calculation effort and accuracy: accurate evaluations have been simplified in order to reduce the complexity of calculations and the required memory, consequently, a reduction of the accuracy is obtained. In (Guerin, 1991), the convolution approach is first applied solely as a binomial distribution over homogeneous sources. Later, the Gaussian distribution is proposed as an approximation to the exact value. Cell Loss Probability evaluation (individual CLR): normally, different classes of traffic are segregated to different VPs. Therefore, all individual connections have the same QOS. Nevertheless, it may be more efficient to transport different classes of traffic by the same VP; then, connections on the same VP have different CLR, and, thus, imply different QOS for different classes. This individual CLRi for each class of traffic is difficult, or impossible, to obtain. 


\subsection{Experiments of Bandwidth Allocation based on analysis}

In the next stage of the work, experiments have been carried out in order to compare the behaviour of different bandwidth allocation approaches for a set of scenarios. Fluid-flow, Linear Approximation and Gaussian are contrasted to the Convolution Approach.

Convolution results are compared to Linear, Fluid-Flux and Gaussian approximations. They have been evaluated in the following manner:

- Linear method: the maximum number of sources that the link can transport $\mathrm{Nj}$ is evaluated by an exact method. The effective bandwidth is $\mathrm{C} / \mathrm{Nj}$ for the $\mathrm{j}$-type sources. Similar procedure is applied to the remaining types. In this case, it is necessary to derive its value off-line; this may be achieved either through analysis or through simulation and experimentation.

- Fluid-Flow model: this approximation has good accuracy when either the number of connections is small or the actual total equivalent capacity is reasonably close to the overall mean rate. An approximation presented in (Guerin, 1991) is used.

- Gaussian allocation approach: this method is based on the assumption that the distribution of the required bandwidth of the existing calls can be approximated by a normal distribution with the same mean and variance. That allows the use of standard approximations to estimate the tail of the bit rate distribution. Formula (6) has been used for this approach.

- Convolution Approach. An exact evaluation of the instantaneous rate on the link is obtained. Utilising the bufferless assumption, Convolution is used for Bandwidth Allocation. In section 4, a detailed study is presented.

All methods calculate a demanded bandwidth in order to ensure a pre-set upper bound for cell loss ratio.

Homogeneous traffic experiments: for these experiments a set of 50 On-Off sources has been analysed. These sources have a mean burst period equal to 100 $\mathrm{ms}$; and the maximum PC allowed is $10^{-5}$.

Fig. 1 shows the demanded bandwidth (y-axis) evaluated by both: Fluid-Flow and Convolution. The number of sources is 50; and each connection has a peak rate equal to $4 \mathrm{Mb} / \mathrm{s}$ The utilisation of each source varies from $10 \%$ to $80 \%$ (x-axis). The fluid-flow model is evaluated for different buffer sizes $(b=0.01,1,2$ and 3 Mbit), $b=0.01$ means in fact small buffers, no differences have been obtained for buffer size up to 128 cells. 


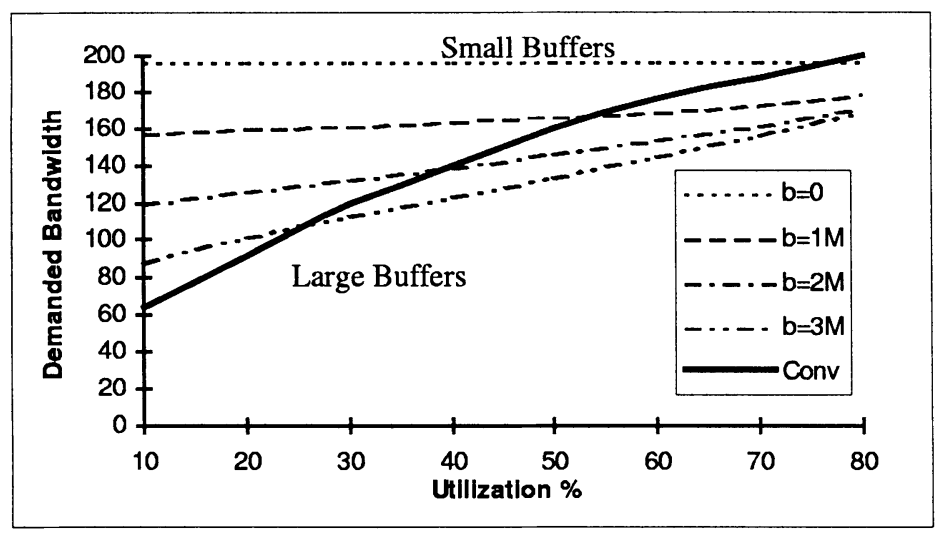

Fig. 1 Demanded Bandwidth vs. utilisation

For source utilisation higher than $25 \%$ and with a buffer size of $3 \mathrm{Mbit}$ the required capacity evaluated by convolution is greater than the required for the Fluid-Flow approximation. Furthermore, when the size of the buffer is 1 Mbit, the same effect occurs when the source utilisation is higher than $55 \%$. When using small buffers, the convolution approach always gives more accurate results. Note that small buffers are used in our study to limit maximum cell delay and jitter. Consequently, Fluid-Flow approximations will not be taken into account in the following experiments.

On the other hand, the convolution approach always evaluates a more accurate demanded Bandwidth than the Gaussian model. From $10 \%$ to $40 \%$ overestimation is observed with the Gaussian model compared to Convolution, for 80 $\%$ of source utilisation.

Heterogeneous traffic experiments. Several experiments with mixed traffic are now presented. On the $y$-axis the demanded bandwidth is shown and on the $\mathrm{x}$-axis all the possible combinations of traffic are presented. The source characterisation has been chosen in the GMDP model. The following table shows the characteristics of the used sources.

\begin{tabular}{cccccc} 
& \multicolumn{2}{c}{ State 0 } & & \multicolumn{2}{c}{ State 1 } \\
\cline { 5 - 6 } \cline { 5 - 6 } & $\begin{array}{c}\text { Rate } \\
\text { (Mbits) }\end{array}$ & Prob & & $\begin{array}{c}\text { Rate } \\
\text { (Mbits) }\end{array}$ & Prob \\
A2 & 0.4 & 0.625 & & 2 & 0.375 \\
B2 & 2 & 0.625 & & 10 & 0.375 \\
C2 & 6 & 0.625 & 30 & 0.375 \\
& & & & &
\end{tabular}

Table 1 Source description

When two types, B2 and C2, are mixed, and the maximum number of connections is 7 and 42 respectively. In this scenario the Gaussian approximation is also 
accurate, except for combinations of traffic that correspond to a small number of connections.

In the following experiment, three types are mixed: $\mathrm{A} 2, \mathrm{~B} 2$ and $\mathrm{C} 2$, the maximum number of connections is 15,10 and 3 respectively.

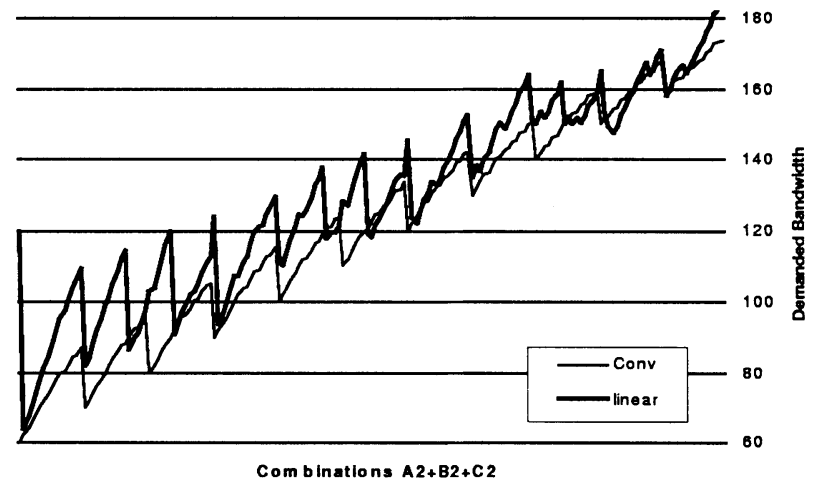

Fig. 2 Demanded Bandwidth for heterogeneous traffic

For simplicity, only combinations with one $\mathrm{C} 2$ connection and varying $\mathrm{B} 2$ and $\mathrm{A} 2$ types of traffic are plotted Fig. 2, since the remaining combinations show similar behaviour. The linear approximation has a changing behaviour that tends to be conservative. Differences up to $50 \%$, case of low number of connections, can be observed.

Using small buffers, a premise in this study, the convolution approach gives always more accurate results than Fluid Flow approximations. The required capacity evaluated by convolution is normally less than the one required for the Gaussian assumption. In the presence of bursty traffic, utilisation less than $20 \%$, the Gaussian evaluation is too optimistic. Guerin et al. (Guerin, 1991) propose using Fluid-Flow approximations in this situation. Moreover, for small buffers the required bandwidth is perceptively higher than the Gaussian. Moreover, it is not clear how to define this situation. This effect is unwanted because the actual cell loss may be greater than the evaluated cell loss ratio and, consequently, the QOS requirements established for the user could not be guaranteed. Finally, the linear approximation varies from pessimistic to optimistic depending on the mixture of traffic.

\section{THE PC AS BW ALLOCATION DECISION PARAMETER}

\subsection{ATM network model}

There are some services for which the QOS has real time constraints (i.e. interactive services), for delay; these services are considered in the standards as rtVBR bearer capabilities. Therefore, very large buffers cannot be introduced and buffer dimensioning is carried out taking into account the cell level contention. Also, suitable buffer sizes can be selected to ensure that the maximum cell delay is less than a pre-specified limit (Yang, 1993). Under that premise, Cell Loss Ratio (CLR) is the major relevant Parameter of QOS. 
Therefore, the buffer size of the statistical multiplexer is assumed to be small, in order to guarantee an acceptable maximum delay (e.g. 50 cells corresponds to 140 $\mu \mathrm{s}$. , link rate $=150 \mathrm{Mbit} / \mathrm{s})$. LAN interconnect is an important example of a service which requires both a low loss rate and a low end-to-end delay (Wright, 1989) due in the main to time-outs in the LAN protocols.

\subsection{The Probability Of Congestion.}

To work with small buffers implies that the traffic bursts cannot always be saved in the buffer. Therefore, the burst length is irrelevant because the majority of cells will be lost. On the other hand, it is not likely that users will be able to supply information about the burst length at connection set-up.

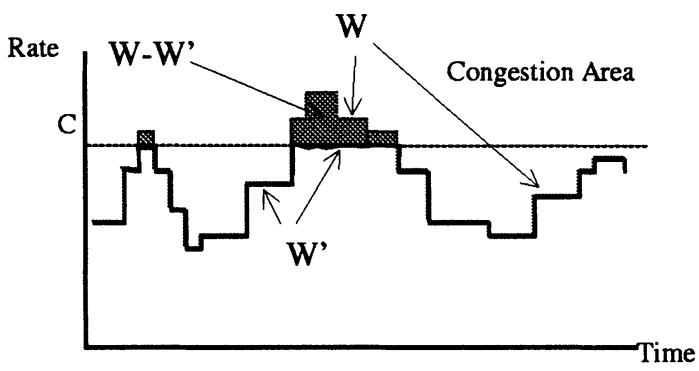

(a) Time scale

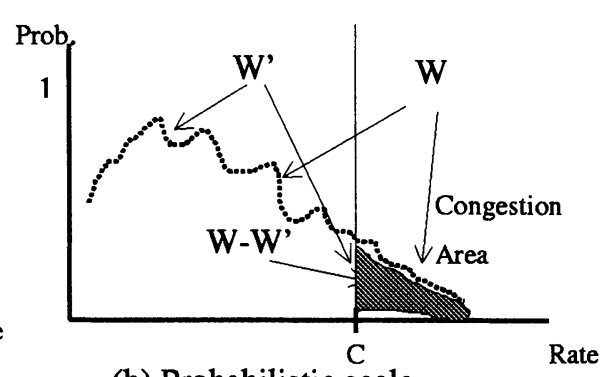

(b) Probabilistic scale

Fig. 3 Congestion in an ATM link

W and $W^{\prime}$ represent the offered and the carried traffic respectively. Under the bufferless assumption W-W' means the lost traffic. Fig. 3 (a) shows the instantaneous aggregated rate of all sources connected against time. Fig. 3 (b) shows the probability associated to a given instantaneous aggregated bit rate of all sources; all situations corresponding to rates greater than $\mathrm{C}$ (at right of $\mathrm{C}$ ) are in a congestion state.

The probability of congestion (PC) is the sum of probabilities corresponding to rates greater than $\mathrm{C}$, which is the shadow area. The probability of congestion does neither state how many cells are lost nor the duration of the congestion state, but only that there is cell loss (Iversen, 1991). The load region admissible is approximated using parameters such as; the mean load, the congestion probability, and the ratio of cells exceeding the link capacity for the total cell stream and for each individual connection. For arbitrary mixes, the load of the link may provide only little information about the cell loss probabilities. Cell losses are quite likely if the bandwidth required at the burst level exceeds the capacity of the link. These events are taken into account by the PC.

$$
P C(Y)=P(Y>C)=\sum_{R>C} P(Y=R)
$$

$\mathrm{Y}$ means the bandwidth distribution in terms of instantaneous rate, and $\mathrm{C}$ is the rate of the link. However, the PC does not give any information about the number of cells lost in case of congestion unlike Cell Loss Ratio (CLR). In a short congestion 
state, all cells may be buffered with no cell losses occurring. Nevertheless, when a burst's duration is longer than the size of the buffer, then almost all cells exceeding the link capacity are lost. In this case, the relation between PC and CLR is approximated by:

$$
C L R(Y)=\frac{\sum_{R>C}(R-C) P(Y=R)}{E(Y)}
$$

If the buffer size is sufficient for cell contention, the evaluated CLR provides an upper bound to the total cell loss probabilities. The PC model is a stationary approximation, in other words, a probabilistic scheme.

\section{BANDWIDTH ALLOCATION BASED ON THE PROBABILITY OF CONGESTION}

In this section, different methods to obtain Probability of Congestion (PC) on a link based on the convolution function are presented. The cost involved in the PC calculation is also analysed.

\subsection{The Formula-Based Convolution Approach}

This section contains the calculation of the bandwidth requirements of the superposition of several sources. This approach is based on the well-known expression of the convolution procedure denoted by:

$$
Q=Y^{*} X
$$

which is evaluated by the following expression:

$$
P(Y+X=b)=\sum_{k=0}^{b} P(Y=b-k) P(X=k)
$$

where $Q$ is the bandwidth requirement of all established connections including the new connection; $\mathrm{Y}$ is the bandwidth requirement of the already established connections; $\mathrm{X}$ is the bandwidth requirement of a new connection, and $\mathrm{b}$ denotes the instantaneous required bandwidth. This function is expressed as the probability that all traffic sources together are emitting at a given rate $b$. We take into account that the evaluated offered load is not the link load itself, but the load generated by all the traffic sources intended to be carried by the link. The link carries this load in non-congestion state only.

The direct application of the expression (6) in order to evaluate the convolution is difficult in practice. In order to obtain the probabilistic distribution on the link, a vector containing all possible rate-probability pairs is defined; this vector is called System Status Vector (System-SV). To obtain the complete System-SV, the following process is carried out: whenever a new connection demand arrives the System-SV must be updated; the corresponding Source-SV is used to do this update, and for each old System-SV element a set of new System-SV elements is generated. The rate of each new element is the sum of the existing rate and the rate corresponding to the state of the new source. The probability of each new element 
is the product of the existing probability and the new probability corresponding to the state of the new source. By using this method N-1 convolutions are needed for each new connection. The expression (5) can be re-written as (Iversen, 1990):

$$
\mathrm{Qn}=\mathrm{Qn}-1 * \mathrm{Xn} ; \mathrm{n} \in\{1,2, \ldots, \mathrm{N}-1)
$$

Considering $Q_{0}=X_{0}$. Clearly, we should carry out N-1 convolutions to obtain the global distribution. At any point in time, an ATM link can carry several thousand connections. As described in a previous section, when a connection is accepted, the new connection is convoluted with the global steady-state probabilities of all existing connections. When a connection terminates it would be preferable to deconvolute all existing connections. So the feasibility of the deconvolution operation is very important. The problem is that the global steady state has now changed; this means that some previously calculated values are lost. The reason for this is that a) the accumulation of probabilities, corresponding to the same rate and b) very small intermediate values, are not considered. Furthermore, by not truncating the state, the space required for storage increases; the number of arithmetic operations further increases. These aspects, relating to accuracy and cost, are more widely developed in the following sections.

\section{Drawbacks}

In (Iversen, 1990 and 1991), (Kroner, 1990), (Kaltenmorgen, 1992), (R1022, 1990), (Del 122, 1991), (Miah, 1994) and (Ramalho, 1994) some limits of the Convolution Approach are pointed out. High cost in terms of storage requirements. Note That a huge amount of memory storage $M$ is required by the System-SV. This requirement increases dramatically with the number of connections $\mathrm{N}$ and source states $T_{j}$. High cost in terms of calculation. The computing time depends on the complexity of the distribution itself. The time needed for the convolution increases with the number of states per connection. Individual QOS. The evaluated link status using a convolution approach makes no distinction between individual connection. Thus, the individual QOS, in terms of cell loss, for each type of source is not available.

\subsection{The Enhanced Convolution Approach (ECA)}

To overcome the drawbacks associated with the PC calculation, a new method of evaluation is proposed: the Enhanced Convolution Approach (ECA). In this method, the multi-nomial distribution function is first applied to groups of the same type of sources, and the global state probabilities are finally evaluated by convolution of the partial results obtained from the different existing groups of sources.

The state of the link can be expressed as a function of the number of active connections of each service type $\left(n_{0}, n_{1}, \ldots n_{j}, \ldots, n_{L-1}\right)$. This is because the state of the link depends only upon a service's occupancy. First the multinomial function is applied to homogenous sources producing intermediate results. Finally, from these intermediate results a final result is obtained by convoluting one element of a given class of traffic with one from each of the other classes. This process is called multiconvolution in this study. 


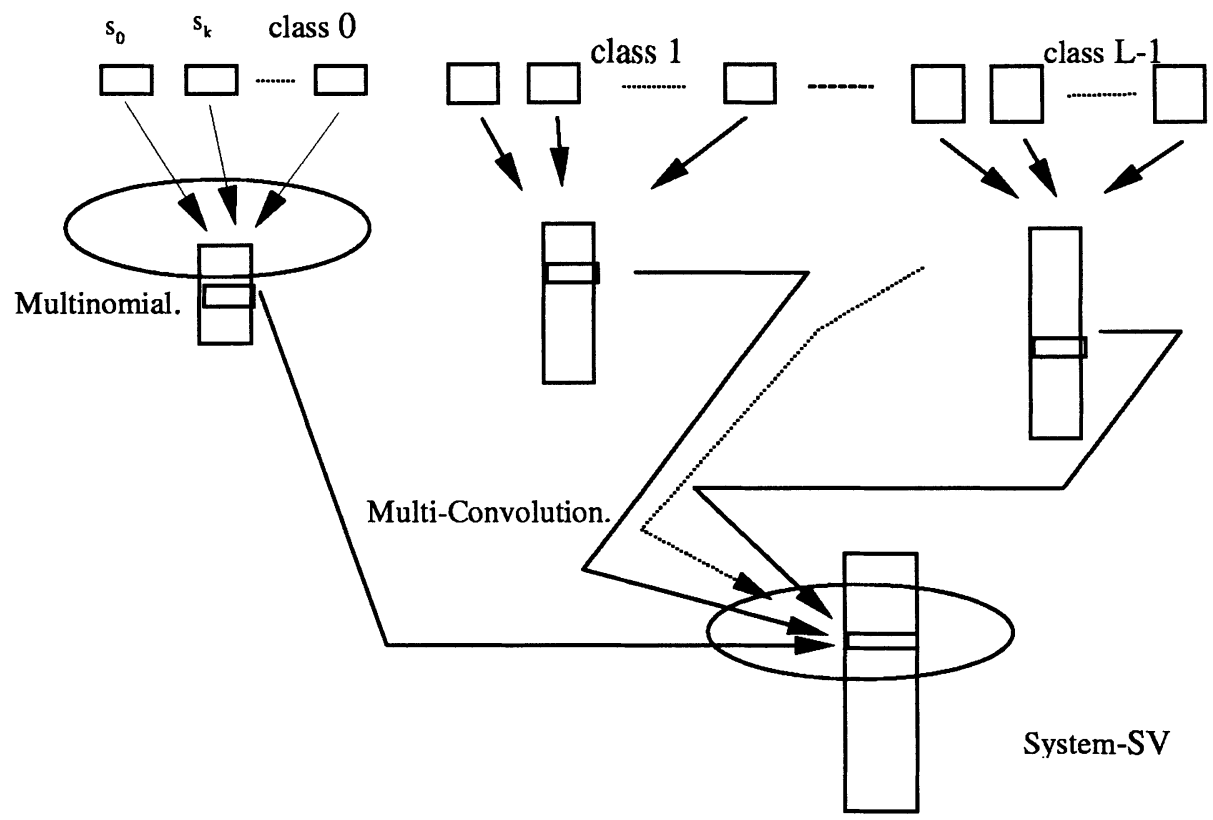

Fig. 4 Overview of the method.

\section{The Multinomial Distribution Function (MDF)}

After computing the convolution the same rate may appear more than once in the System-SV. Which elements are repeated? How many times? This is expressed as a probability corresponding to the MDF, see (Ash, 1969) and (Hogg, 1989).

$\mathrm{P}\left(\mathrm{n}_{0}, \mathrm{n}_{1}, \ldots, \mathrm{n}_{\mathrm{T}-1}\right)=\frac{N !}{n_{0} ! n_{1} ! \ldots n_{T-1} !} p_{0}^{n_{0}} \cdot p_{1}^{n_{1}} \ldots p_{T-1}{ }^{n_{T-1}}$

The corresponding rate can easily evaluated by the following expression:

$$
\mathrm{r}\left(\mathrm{n}_{0}, \mathrm{n}_{\mathrm{t}}, \ldots, \mathrm{n}_{\mathrm{T}-1}\right)=\sum_{i=0}^{T-1} n_{i} \cdot r_{i}
$$

Note that the probability of each source being in state $s_{i}$ is independent of the probability of the other source states.

To evaluate the ECA, some data structures are necessary at this phase. For $\mathrm{N}$ connections of the same type, there is an associated Sub-Matrix (SMX). 


$$
\operatorname{SMX}(N)=\left[\begin{array}{cccc}
n_{0,0} & n_{0,1} & n_{0, j} & n_{0, T-1} \\
\ldots & \ldots & \ldots & \ldots \\
n_{r, 0} & n_{r, 1} & n_{r, j} & n_{r, T-1} \\
n_{M-1,0} & n_{M-1,1} & n_{M-1, j} & n_{M-1, T-1}
\end{array}\right]=\left[\begin{array}{c}
S M X_{0} \\
\ldots \\
S M X_{r} \\
\ldots \\
S M X_{M-1}
\end{array}\right]
$$

$\mathrm{SMX}_{\mathrm{r}}$ is the generic row of SMX. The number of columns is equal to the number of source rates T. SMX stores the distribution of the connections from each state. The system load density function is obtained directly from the sub-matrix using the MDF expression.

\section{The multi-convolution procedure}

When there are different types of sources $j$ (heterogeneous traffic), it is necessary to 'convolute' between all source types. To store all possible combinations relating to the system state, a System Status Matrix (SSM) is defined. The generic elements of the SSM, namely the general system status rows SSM $_{\mathrm{r}}$, are generated each by concatenating all possible combinations between the different sub-matrices rows $\mathrm{SMX}_{\mathrm{r}}$, associated with the $\mathrm{L}$ different $\mathrm{j}$-types of sources $(\mathrm{j}=0,1, \ldots, \mathrm{L}-1)$.

and from (12)

$$
S S M_{r}=<S M X_{r_{0}, o}, \ldots, S M X_{r_{L-1}, L-1}>\quad \forall \mathrm{r}=0, \ldots, \mathrm{M}_{\mathrm{j}-1}
$$

$$
S S M_{r}=<n_{r_{0}, o}, \ldots, n_{r_{0}, S_{j}-1}, \ldots, n_{r_{L-1}, L-1}, \ldots, n_{r_{L-1}, L_{j}-1}>
$$

Based on the ECA algorithm, grouping connection in types, the following expression for the cell loss probability of the type-j traffic proposed:

$$
C L R_{j}=\frac{\sum_{W>C} \frac{W_{j}}{W}(W-C) P(Y=W)}{E\left(Y_{j}\right)}
$$

$\mathrm{Wj}$ is the rate offered by all type-j traffic when the instantaneous offered rate on the link is $L$ and $E\left(Y_{j}\right)$ is the mean rate of all traffic of type-j. Both terms are easily obtained during the evaluation of PC based on the ECA algorithm. This is the evaluation of the CLR to a type- $j$ traffic.

This method is widely detailed in (Fabregat, 1995) and (Marzo, 1993).

\section{CAC BASED ON THE PROBABILITY OF CONGESTION}

This section is focused on reasonable real-time processing, storage requirements as well as the maximisation of statistical multiplexing gain. Obviously, all the methods studied intend to guarantee QOS for all established connections.

\subsection{Implementation Issues}

In order to evaluate the $\mathrm{PC}$, it is not necessary to completely evaluate the entire distribution of the instantaneous rate. Using ECA it is possible to evaluate a part of the statistical distribution. The techniques presented next are attempted to evaluate 
only the major relevant part of the system state: Congestion. All five cut-off improvements can be implemented simultaneously.

Link Capacity Cut-off: the calculation of probability is only carried out in cases where the associated rate exceeds the bandwidth provided $\mathrm{C}$. The associated probabilities with rates smaller than $\mathrm{C}$ are not calculated (i.e. the MDF is not evaluated).

Probability of Congestion Cut-off. The PC of the system is compared with a previously set value in order to guarantee the specific QOS. Therefore, if during the process of calculation the current PC exceeds a pre-set value, the process is stopped and the calculation cost is thus reduced.

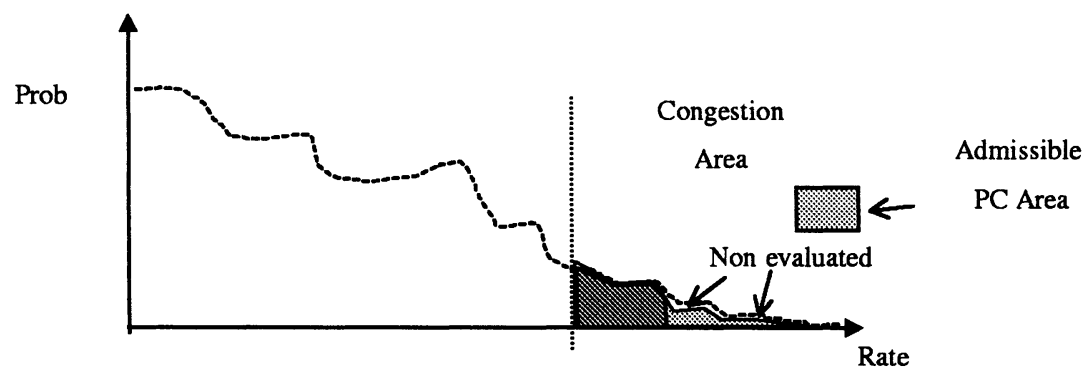

Fig. 5 Probability of Congestion Cut-Off

Partial Sorting Cut-off. Furthermore, in each System Status Matrix (SSM) the rows generated are not examined in an arbitrary order, but are graded according to rate, so when the pre-set minimum rate $\mathrm{C}$ is reached, the process is terminated and a further saving computation time is achieved.

Small probabilities Cut-off. When the probability obtained by the Multinomial Distribution Function, is less than a pre-set threshold value this result can be ignored. Therefore, the corresponding element is not stored. This threshold value depends on the maximum congestion probability and on the number of connections.

Grouping states Cut-off. For those classes with a large number of connections the majority of information enclosed in the sub-matrix may be summarised by grouping states. This mechanism could be applied independently to each class of sources before evaluating the second phase in the calculation of PC.

\subsection{Cost Experiments}

All experiments have been based on a General Modulated Deterministic Process (GMDP) source model. The GMDP model describes the behaviour of a traffic source at cell and burst level. The number of states for j-type sources is $\mathrm{Sj}$. In each state $\mathrm{i}$ (with $\mathrm{i}=0,1, \ldots, \mathrm{Sj}-1$ ), during the corresponding sojourn time SojTj,i, cells are sent with regular inter-arrival times (constant rate rj,i,)

The evaluation cost is measured in terms of a number of different metrics: Storage requirements are measured in elements, each element has to store a rate and a probability. The time parameter corresponds to CPU time and is expressed in normalised time (seconds in the presented experiments). Sorting techniques are required to put the partial status vector in order. The quick sort algorithm is used 
when necessary. The cost is expressed as $\mathrm{x} \cdot \log (\mathrm{x})$, where $\mathrm{x}$ is the number of elements to be arranged. Calculation cost is expressed as a normalised combination of additions and products

The computational efficiency has been measured for both the formula based (basic) convolution and the ECA. A comparison in time is shown in the 'speed-up' column of the following table, the time obtained for the basic convolution is set to 1 ; the evaluations for the enhanced convolution approach are normalised to this value.

Two types of sources (60 and 75 connections) are multiplexed in an $600 \mathrm{Mbit} / \mathrm{s}$ ATM link. In the presence of only one class of traffic the application of the ECA increases the speed-up factor.

\begin{tabular}{|c|c|c|c|c|c|c|c|c|}
\hline & $\mathrm{Mval} / \mathrm{Gr}$ & PC & CLP & time & sp-up & storage & sorts & cost \\
\hline Bas: & & $2 \mathrm{E}=4$ & $1.2 \%$ & 486 & & 13252 & 174880 & 1.2 \\
\hline ECA & - & $2 \mathrm{E}-4$ & $1.2 \mathrm{E}-5$ & 0.95 & 3 & 3439 & 5 & 9398 \\
\hline $\mathrm{ECA}$ & 1.E-7/- & $2 \mathrm{E}-4$ & $1.1 \mathrm{E}-5$ & 0.04 & 122 & 709 & 1 & 327 \\
\hline+ & $-/ 3$ & $"$ & $"$ & 0.12 & 4. & 1147 & 5 & 1095 \\
\hline \multirow[t]{3}{*}{ Cut off } & $-/ 5$ & $2 \mathrm{E}-4$ & $1.2 \mathrm{E}-5$ & 0.06 & 81 & 689 & 5 & 432 \\
\hline & $1 \mathrm{E}-8 / 3$ & $2 \mathrm{E}-4$ & $1.2 \mathrm{E}-5$ & 0.02 & 248 & 372 & 1 & 97 \\
\hline & $1 E-08 / 5$ & $2 E-4$ & $1.2 E-5$ & 0.01 & 4860 & 163 & 1 & 70 \\
\hline
\end{tabular}

Table 2 Cost results for heterogeneous traffic

Sorting is the dominant factor for the formula-based convolution, whereas cost evaluation is the dominant factor for the enhanced convolution. Another conclusion is the efficacy of the small probabilities cut-off. The first direct implication is the reduction in the storage requirements. Moreover, this reduction in the intermediate vectors implies a rise from 5 up to 500 times faster in the carried out experiments.

\section{CAC EXPERIMENTS}

This Section discusses different aspects of the behaviour of cell streams in an output buffer corresponding to an ATM link and is illustrated by experimentation. CAC experiments relating to Fuzzy logic and $(M+1)$-MMDP approaches are presented. Measurements in an ATM test bed are also included.

The experiments described in this section refer to a single ATM link and the QOS is expressed in terms of cell loss at the output buffer of an ATM switch. The traffic sources are VBR sources, modelled as On-Off sources described by the peak and mean bit rates and mean burst length. Experiments in homogenous scenarios reveals similar results than for heterogeneous scenarios and have been omitted in this paper.

RACE projects provide ATM test-beds on which measurements and tests can be performed (Kuhn, 1994). This set of experiments enables comparison of the average cell loss results obtained from on-line measurements in the Exploit ATM test-bed in Basel (R2061/28, 1994) with the cell loss predictions given by both the ECA and FCAC approaches for homogeneous and heterogeneous traffic scenarios 
on a single ATM link. Although FAC attempts to predict the maximum cell loss ratio per connection instead of the average cell loss ratio for the aggregate traffic, for the sake of comparison with the results obtained from on-line measurements, FCAC was trained to predict the average cell loss ratio instead. In Table 3 the traffic sources used for the comparison experiments are described. The link capacity considered is $155.52 \mathrm{Mbit} / \mathrm{s}$ and the output buffer size is 27 cells.

\begin{tabular}{ccccc} 
Traffic type & $\begin{array}{c}\text { Peak Rate } \\
(\text { Mbit/s) }\end{array}$ & $\begin{array}{c}\text { Mean Rate } \\
\text { (Mbit/s) }\end{array}$ & & $\begin{array}{c}\text { Mean Burst L. } \\
\text { (cells) }\end{array}$ \\
\cline { 1 - 1 } A31 & 31.1 & & 6.22 & 1467 \\
B31 & 7.78 & & 3.89 & 917 \\
C31 & 1.94 & 0.97 & 229 \\
\hline
\end{tabular}

\section{Table 3 Characteristics of the traffic sources}

4 A31 connections are mixed with 6 to 24 connections of B31 traffic in experiment B5. 4 A31 connections are mixed with 24 to 100 connections of C31 traffic in experiment B6. We can see in both experiments, that the cell loss results obtained by ECA and FCAC are very similar to those obtained by measurements in the Exploit test-bed.

Considering that the buffer size used in the ATM test-bed experiments is small (27 cells), it is not surprising that the cell loss predicted by the ECA is so close to the cell loss measured values. This also explains the slight difference between the measurements curve and the convolution curve as some of the generated cells can be stored in the server output buffer, and, therefore, a more optimistic cell loss is obtained (see measurements curve).
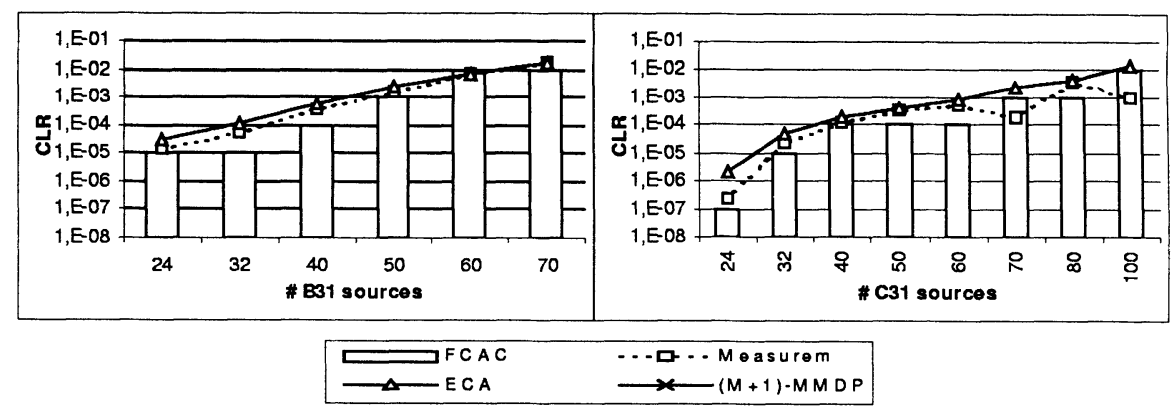

Fig. 6 (a)Experiments $B 5$ and $(b)$ experiments $B 6$

The prediction results based on the convolution approach have been obtained using the ECA algorithm without considering optimisations. The cut-off mechanisms and other improvements biased to achieve a fast evaluation of the CLR can only be used for CAC. Anyway, for the experiments presented, the time required for the evaluation was negligible. 


\section{CONCLUSIONS}

In this paper the utilisation of the Probability of Congestion (PC) as a bandwidth allocation decision parameter has been presented. The validity of PC utilisation is compared with QOS parameters in small buffer environments when only the CLR parameter is relevant.

To overcome the drawbacks of the formula-based Convolution Approach, a new method of evaluation is analysed: the Enhanced Convolution Approach (ECA). Sorting is the dominant cost factor for the formula-based convolution, whereas calculation is the dominant cost factor for the ECA. With reference to the cut-off mechanisms presented, the major conclusion is the efficacy of the low probability cut-off. This mechanism implies is a reduction in the storage requirements and a reduction of the evaluation time. The ECA also enables the computation of the Individual Cell Loss Ratio for each $\mathrm{j}$-class of traffic.

It can be summarised that the convolution algorithm seems to be a good solution for CAC in ATM networks with relatively small buffers. If the source characteristics are known actual cell loss ratio can be accurately estimated. Furthermore, this estimate is always conservative, allowing the provision of the network performance guarantees. We can also conclude that by combining the ECA method with cut-off mechanisms, utilisation of ECA in real-time CAC environments as a single level scheme is possible.

Source modelling for more realistic traffic is now an open issue. The ECA utilisation does not take account of temporal references (burst length), so that the source parameterisation is simplified. On the other hand, more realistic traffic, such as VBR video sources, can be modelled as sources with more than two associated states. ECA can also be use with these new models.

By simple analysis of the ECA evaluation (see Fig. 4), a parallelisation of the ECA algorithm is a further step (the evaluation of each sub-matrix corresponding to each class of traffic can also be obtained in parallel).

\section{REFERENCES}

Ash, R. (1969). Basic Probability Theory. John Wiley \& Sons.

Bolla, R. Davoli, F. and Marchese, M. (1997) Bandwidth Allocation and Admission Control in ATM Networks With Service Separation. IEEE Comunications Magazine. Vol 35 No. 5 130-137.

Castelli, P. Cavallero, E. And Tonietti, A. (1991) Policing And Call Admission Problems in ATM Networks. Teletraffic and Datatraffic, ITC-13 847-852.

Del. 122 (1991). Progress Report on the CAC and Policing Experiments. COST.

Decina, M. And Toniatti, T. (1992) Bandwidth Allocation and Selective Discarding for VBRV and Bursty Data Calls in ATM Networks. International Journal of Digital and Analog Communications Systems.

Marzo, J.L. Domingo, J. Fabregat, R. and Sole-Pareta, J. (1995) Dynamic Routing Based on a Single Parameter: Link Congestion Probability. High Performance Networking VI. IFIP. Chapman \& Hall. 307-318

Gallasi, G. Rigolio, G. and Fratta, L. (1989). ATM: Bandwidth Assignment and Bandwidth Enforcement Policies. Globecom 89. 
Guerin, R. Ahmadi, H. and Naghshineh, M. (1991) Equivalent Capacity and its Application to Bandwidth Allocation in High-Speed Networks. IEEE Journal On Selected Areas In Communications, Vol 9, N7, 968-98.

Hogg, R. Allen, V. And Craig T. (1989) Introduction to Mathematical Statistics. Maxwell MacMillan Int Ed.

Handel, R. Huber, M. N. And Schroder, S. (1994) ATM Networks. Concepts, Protocols and Applications. Addison-Wesley.

Hee-Jeon, Y. and Viniotis, I. (1993) Feasibility of Performance Objectives in ATM Network Nodes with MMPP Arrival Processes. Modeling and Performance Evaluation Of ATM Technology (C-15). 119-134.

Hui, J. Y. (1988) Resource Allocation for Broadband Networks. IEEE Journal on Selected Areas in Communications, Vol 6 N 9, 1598-1608.

Iversen, V.B. and Yun Liu. (1990) The Performance of Convolution Algorithms for Evaluating the Total Load in an ISDN System. Ninth Nordic Teletraffic Seminar, Norway.

Iversen, V.B. and Bohn-Nielsen, A. (1991) Statistical Multiplexing In ATMNetworks. RACE 1022.

Kaltenmorgen, B. (1992) FIDBP. Connection Acceptance Control. BAF-ProjectFIDBP-92301-Cd-Cc/A.

Kleinvewillinghöfer-Kopp, R. and Kaltenmorgen, B. (1991) Connection Acceptance Control In ATM Networks. Studie des Forschunginstitus der DBP Telekom, Forschungsbereich 5 'Vermittlung und Netze'

Kroner, H. (1990) Algorithms For Call Acceptance Control Published At ICC'89Basic Propierties And Preliminary Study. RACE 1022. UST_123_026_CD_CC.

Kuhn, P.J. (1994). Performance Modelling and Traffic Engineering For Broadband Communication Networks. North Holland Broadband Comm.

Marzo, J.L. Fabregat, R. Domingo, J. and Sole-Pareta, J. (1993). Fast Calculation Of The Cac Convolution Algorithm Using The Multinomial Distribution Function. UK TT Symposium. Performance Engineering In Telecommunications Networks. BT Laboratories Ipswich UK.

Miah, B. and Scharf, E. (1994) A Real Time Management System for the TwoLevel CAC Algorithm. 11 UK Teletraffic Symposium. Cambridge.

Miyao, Y. (1993) Bandwidth Allocation in ATM Networks that Guarantee Multiple QOS Requirements. ICC93, 1398-1403.

Ohta, S. and Sato, K. I. (1992) Dynamic Bandwidth Control of the Virtual Path in an Asynchronous Transfer Mode Network. IEEE Transaction on Communications, Vol. $40, \mathrm{~N}^{\circ} .7$.

R1022. (1990) Updated Results of Traffic Simulation of the Policing Experiment. Technology For ATD.

Race 2061 (1994) Exploit, Results Of Experiments On Traffic Control Using Real Aplications. Deliverable 28.

Ramalho, M. F. and Scharf, E.(1994). Fuzzy Logic Based Techniques for CAC in ATM Networks. 11 UK Teletraffic Symposium. Cambridge.

Saito, H. (1992) CAC in an ATM Network using Upper Bound of Cell Loss Probability. IEEE Transactions on Communications. 40, 1512-1521. 
Wright, D. J. and Michael T. (1989) A Characterization of Telecommunication Services in the 1990's. Infocom 89. 624-631.

Yang, T. and Li, H. (1993) Individual Cell Loss Probabilities and Backgroung Effects in ATM Networks. IEEE ICC33, 1373-1379.

Yang, T. and Tsang, H. K. (1995). A Novel Approach to Estimating the CLP in an ATM Multiplexer Loaded with Homogeneous On-Off Sources. IEEE Transaction on Communications.

\section{BIOGRAPHY}

Jose-Luis Marzo is an Associate Professor of Computer Science and Communications at the Universitat de Girona. He received the engineering degree in Computer Science (1989) and the Ph.D. degree (1997). Since 1988 he is teaching at the Electronics and Computer Architecture Department. His research topics are Broadband Communications and Applications. Since 1993 he has participated in Spanish Broadband projects. He is IEEE member.

Jordi Domingo-Pascual is an Associate Professor of Computer Science and Communications at the Universitat Politècnica de Catalunya in Barcelona. There, he received the engineering degree in telecommunications (1982) and the Ph.D. Degree in Computer Science (1987). Since 1983 he is teaching at the Computer Architecture Department. His research topics are Broadband Communications and Applications. Since 1988 he has participated in RACE projects, Spanish Broadband projects, and ACTS projects. Since 1995 he is a researcher at the Advanced Broadband Communications Center of the University (CABA).

R. Fabregat is an Associate Professor of Computer Science and Communications at the Universitat de Girona. He received the engineering degree in Computer Science (1989). Since 1990 he is teaching at the Electronics and Computer Architecture Department. His research topics are Broadband Communications and Applications. Since 1993 he has participated in Spanish Broadband projects.

J. Sole-Pareta. Josep Sole-Pareta received his Master's degree in Telecommunication Engineering in 1984, and his Ph.D. in Computer Science in 1991, both from the Universitat Politecnica de Catalunya. Since 1992 he is an Associate Professor with Computer Architecture Department. He has participated in the R\&D Spanish Program for the development of the Broadband Communications. Within the ACTS program, he is participating in MICC, IMMP and INFOWIN projects. His research interests are in B-ISDN, ATM Networks and Personal Communication Systems. He is member of the IEEE and the ACM (Sigcomm).

ACKNOWLEMENTS: This work has been supported by CICYT (Spanish Education Ministry) under contract TIC95-0982-C02-02 and TEL97-1054-C03-03. 